Proceedings of the Edinburgh Mathematical Society (2007) 50, 217-228 (C)

DOI:10.1017/S0013091505000465 Printed in the United Kingdom

\title{
POSITIVE SOLUTIONS FOR NON-RESONANT SINGULAR BOUNDARY-VALUE PROBLEMS WITH A LINEAR TERM
}

\author{
HAISHEN L $\ddot{U}^{1}$, DONAL O'REGAN ${ }^{2}$ AND RAVI P. AGARWAL ${ }^{3}$ \\ ${ }^{1}$ Department of Applied Mathematics, Hohai University, \\ Nanjing 210098, People's Republic of China \\ ${ }^{2}$ Department of Mathematics, National University of Ireland, Galway, Ireland \\ ${ }^{3}$ Department of Mathematical Sciences, Florida Institute of Technology, \\ Melbourne, FL 32901-6975, USA (agarwal@fit.edu)
}

(Received 31 March 2005)

Abstract This paper presents new existence results for the singular boundary-value problem

$$
\begin{gathered}
-u^{\prime \prime}+p(t) u=f(t, u), \quad t \in(0,1), \\
u(0)=0=u(1) .
\end{gathered}
$$

In particular, our nonlinearity $f$ may be singular at $t=0,1$ and $u=0$.

Keywords: non-resonant singular boundary-value problems; positive solution; upper and lower solution 2000 Mathematics subject classification: Primary 34B15

\section{Introduction}

The singular boundary-value problem (BVP) of the form

$$
\left.\begin{array}{c}
-u^{\prime \prime}=f(t, u), \quad t \in(0,1), \\
u(0)=0=u(1),
\end{array}\right\}
$$

occurs in several problems in applied mathematics [1-4]. In this paper we investigate a more general non-resonant singular Dirichlet BVP, namely

$$
\left.\begin{array}{c}
-u^{\prime \prime}+p(t) u=f(t, u), \quad t \in(0,1), \\
u(0)=0=u(1) .
\end{array}\right\}
$$

where $p \in C[0,1], p(t)>0$ for $t \in(0,1)$, and $f:(0,1) \times(0, \infty) \rightarrow R$ is continuous. Notice that $f$ may be singular at $t=0,1$ and $u=0$. We obtain the existence of $C[0,1] \cap C^{2}(0,1)$ non-negative solutions. Of course, by a solution $u$ of the BVP (1.2) we mean $u:[0,1] \rightarrow R$, which satisfies the differential equation in $(1.2)$ on $(0,1)$ and the stated boundary data. 
We will let $C[0,1]$ denote the class of maps $u$ which are continuous on $[0,1]$, with norm $|u|_{\infty}=\max _{t \in[0,1]}|u(t)|$. Let

$$
\begin{aligned}
& M=\left\{h \in C(0,1): \int_{0}^{1} s(1-s)|h(s)| \mathrm{d} s<\infty,\right. \\
& \quad \lim _{t \rightarrow 0^{+}} t^{2}(1-t)|h(t)|=0 \text { if } \int_{0}^{1}(1-s)|h(s)| \mathrm{d} s=\infty \\
& \left.\quad \text { and } \lim _{t \rightarrow 1^{-}} t(1-t)^{2}|h(t)|=0 \text { if } \int_{0}^{1} s|h(s)| \mathrm{d} s=\infty\right\} .
\end{aligned}
$$

The main results of the paper are as follows.

Theorem 1.1. Suppose the following conditions hold.

(H1) There exists a constant $L>0$ such that, for any compact set $K \subset(0,1)$, there is $\varepsilon=\varepsilon_{K}>0$ with

$$
f(t, x) \geqslant L \quad \text { for all } t \in K, x \in(0, \varepsilon] .
$$

(H2) For any $\delta>0$ there exist $h_{\delta} \in M, h_{\delta}(t)>0$ for $t \in(0,1)$ such that

$$
|f(t, x)| \leqslant h_{\delta}(t) \quad \text { for all } t \in(0,1), x \geqslant \delta .
$$

Then problem (1.2) has at least one positive solution $u \in C[0,1] \cap C^{2}(0,1)$. If, moreover, $f(t, \cdot)$ is non-increasing, for each $t \in(0,1)$, then the solution is unique.

Theorem 1.2. Suppose that (H1) holds. Moreover, suppose the following conditions also hold.

(H3) $f(t, x)=q(t) m(t, x)$ with $q>0$ on $(0,1), q \in M$ and $m:[0,1] \times(0, \infty) \rightarrow R$ is continuous with

$$
\begin{aligned}
|m(t, x)| \leqslant g(x)+h(x) \text { on }[ & 0,1] \times(0, \infty), \\
& g>0 \text { continuous and non-increasing on }(0, \infty), \\
& h \geqslant 0 \text { continuous on }[0, \infty) \\
& h / g \text { non-decreasing on }(0, \infty) .
\end{aligned}
$$

(H4) For any $R>0,1 / g$ is differentiable on $(0, R]$ with $g^{\prime}<0$ a.e. on $(0, R]$ and $g^{\prime} / g^{2} \in L^{1}[0, R]$. In addition, suppose that there exists $C>0$ with

$$
\left[1+\frac{h(C)}{g(C)}\right]^{-1} \int_{0}^{C} \frac{\mathrm{d} u}{g(u)}>b_{0}
$$

holding; here

$$
b_{0}=2 \max \left\{\int_{0}^{1 / 2} t(1-t) q(t) \mathrm{d} t, \int_{1 / 2}^{1} t(1-t) q(t) \mathrm{d} t\right\} .
$$

Then problem (1.2) has at least one positive solution $u \in C[0,1] \cap C^{2}(0,1)$. 
Remark 1.3. In [3], the authors consider the BVP (1.2) with $p(t) \equiv 0$ for $t \in[0,1]$ under conditions (H1) and (H2).

Remark 1.4. In [1, p. 186], the authors consider the BVP (1.2) with $p(t) \equiv 0$ for $t \in[0,1]$ under conditions (H1), (H3) and (H4).

Remark 1.5. If $p \in C[0,1], p(t)>0$ for $t \in(0,1)$, then note that

$$
\begin{aligned}
-u^{\prime \prime}+p(t) u & =0, \quad t \in(0,1), \\
u(0) & =0=u(1),
\end{aligned}
$$

has only the trivial solution.

Corollary 1.6. Suppose (H1) and (H2) (or (H1), (H3) and (H4)) hold. Then, for every fixed $\lambda>0$, the problem

$$
\begin{gathered}
-u^{\prime \prime}+\lambda u=f(t, u), \quad t \in(0,1), \\
u(0)=0=u(1),
\end{gathered}
$$

has at least one positive solution $u \in C[0,1] \cap C^{2}(0,1)$.

To conclude this section we look at an example. Consider the BVP

$$
\left.\begin{array}{rl}
-u^{\prime \prime}(t)+\lambda u & =\frac{1}{u^{\alpha}} \quad \text { for } t \in(0,1), \\
u(0) & =u(1)=0,
\end{array}\right\}
$$

where $\lambda \geqslant 0$ and $\alpha>0$.

For this example we cannot apply [3, Theorem 2]. Also it is difficult to demonstrate the conditions (for example $\lambda=2, \alpha=20$ ) [1, Theorem 2.7.7]. However Corollary 1.6 immediately guarantees that (1.4) at least has a solution $u \in C[0,1] \cap C^{2}(0,1)$ with $u(t)>0$ for $t \in(0,1)$ for every fixed $\lambda \geqslant 0, \alpha>0$.

\section{The proof of Theorem 1.1}

From [1, Theorem 1.11.1], we know that

$$
\begin{gathered}
-u^{\prime \prime}+p(t) u=0, \quad t \in(0,1), \\
u(0)=0, \quad u^{\prime}(0)=1,
\end{gathered}
$$

has only one increasing positive solution $e_{1}(t)=t b_{1}(t) \in C[0,1] \cap C^{1}[0,1)$, where $b_{1} \in$ $C[0,1]$ satisfies

$$
b_{1}(t)=1+\frac{1}{t} \int_{0}^{t} \int_{0}^{\eta} \tau p(\tau) b_{1}(\tau) \mathrm{d} \tau \mathrm{d} \eta
$$

Also,

$$
\begin{gathered}
-u^{\prime \prime}+p(t) u=0, \quad t \in(0,1) \\
u(1)=0, \quad u^{\prime}(1)=-1
\end{gathered}
$$


has only one decreasing positive solution $e_{2}(t)=(1-t) b_{2}(t) \in C[0,1] \cap C^{1}(0,1]$, where $b_{2} \in C[0,1]$ satisfies

$$
b_{2}(t)=1+\frac{1}{1-t} \int_{t}^{1} \int_{\eta}^{1}(1-\tau) p(\tau) b_{2}(\tau) \mathrm{d} \tau \mathrm{d} \eta
$$

Let

$$
G(t, s)=\frac{1}{\omega} \begin{cases}e_{2}(t) e_{1}(s), & 0 \leqslant s \leqslant t \leqslant 1 \\ e_{2}(s) e_{1}(t), & 0 \leqslant t \leqslant s \leqslant 1\end{cases}
$$

where

$$
\omega=\left|\begin{array}{ll}
e_{2}(t) & e_{2}^{\prime}(t) \\
e_{1}(t) & e_{1}^{\prime}(t)
\end{array}\right|=\text { const. }>0
$$

It is easy to see that

$$
0 \leqslant G(t, s) \leqslant G(s, s), \quad 0 \leqslant s, t \leqslant 1 .
$$

Consider the two-point BVP

$$
\left.\begin{array}{c}
-u^{\prime \prime}+p(t) u=v(t, u), \quad t \in(0,1), \\
u(0)=a=u(1),
\end{array}\right\}
$$

where $v: D \rightarrow R$ is a continuous function and $D \subset(0,1) \times R$. By a solution $u(\cdot)$ of $(2.3)$ we mean a function $u \in C[0,1] \cap C^{2}(0,1)$ such that $(t, u(t)) \in D$ for all $t \in(0,1)$ and $-u^{\prime \prime}+p(t) u=v(t, u)$ for all $t \in(0,1)$ with $u(0)=a=u(1)$.

Let $\alpha \in C[0,1] \cap C^{2}(0,1)$ satisfy the following conditions: $(t, \alpha(t)) \in D$ for all $t \in(0,1)$ and

$$
\begin{gathered}
-\alpha^{\prime \prime}+p(t) \alpha \leqslant v(t, \alpha), \quad t \in(0,1), \\
\alpha(0) \leqslant a, \quad \alpha(1) \leqslant a .
\end{gathered}
$$

In this case, we say that $\alpha(\cdot)$ is a lower solution of problem (2.3). The definition of an upper solution $\beta(\cdot)$ of problem (2.3) is given in a completely similar way, just by reversing the above inequalities. Also, if $\alpha, \beta \in C[0,1]$ are such that $\alpha(t) \leqslant \beta(t)$ for all $t \in[0,1]$, we define the set

$$
D_{\alpha}^{\beta}:=\{(t, x) \in(0,1) \times R: \alpha(t) \leqslant x \leqslant \beta(t)\} .
$$

We then have the following result.

Theorem 2.1. Let $\alpha$ and $\beta$ be, respectively, a lower solution and an upper solution of problem (2.3) such that

(a1) $\alpha(t) \leqslant \beta(t)$ for all $t \in[0,1]$, and

(a2) $D_{\alpha}^{\beta} \subset D$. 
Assume also that there is a function $h \in M, h(t)>0$, for $t \in(0,1)$, such that

(a3) $|v(t, x)| \leqslant h(t)$ for all $(t, x) \in D_{\alpha}^{\beta}$.

Then problem (2.3) has at least one solution $\tilde{u}(\cdot)$ such that

$$
\alpha(t) \leqslant \tilde{u}(t) \leqslant \beta(t) \quad \text { for all } t \in(0,1) .
$$

Proof of Theorem 2.1. The proof follows the argument in [3]. For convenience, we sketch it here.

First of all we define an auxiliary function

$$
v^{*}(t, x):= \begin{cases}v(t, \alpha(t)), & x<\alpha(t), \\ v(t, x), & \alpha(t) \leqslant x \leqslant \beta(t), \\ v(t, \beta(t)), & x>\beta(t) .\end{cases}
$$

By (a2) and the definition of $v^{*}$ it can easily be checked that $v^{*}:(0,1) \times R \rightarrow R$ is continuous. From (a3) we have

$$
\left|v^{*}(t, x)\right| \leqslant h(t) \quad \text { for }(t, x) \in(0,1) \times R .
$$

Consider now the problem

$$
\left.\begin{array}{c}
-u^{\prime \prime}+p(t) u=v^{*}(t, u) \quad \text { for } t \in(0,1), \\
u(0)=a=u(1) .
\end{array}\right\}
$$

It can easily be verified that the Green function of the problem

$$
\begin{gathered}
-u^{\prime \prime}+p(t) u=v^{*}(t, u) \quad \text { for } t \in(0,1), \\
u(0)=0=u(1)
\end{gathered}
$$

is the function $G:[0,1] \times[0,1] \rightarrow[0, \infty)$ given by $(2.1)$. Define the operator $T$ by

$$
(T u)(t):=a+\int_{0}^{1} G(t, s) v^{*}(s, u(s)) \mathrm{d} s .
$$

From (2.4) and the definition of $v^{*}$ it follows that

$$
T: X=C[0,1] \rightarrow X
$$

is defined, continuous and that $T(X)$ is a bounded set. Moreover, $u \in X$ is a solution of (2.5) if and only if $u=T u$.

The existence of a fixed point for the operator $T$ will now follow from the Schauder fixed-point theorem if we show that $T(X)$ is relatively compact.

Let $t \in(0,1)$. Then, using $(2.4)$, we have

$$
\left|\frac{\mathrm{d}}{\mathrm{d} t} T(u)(t)\right| \leqslant \frac{C_{1}}{\omega}\left[\int_{t}^{1} e_{2}(s) h(s) \mathrm{d} s+\int_{0}^{t} e_{1}(s) h(s) \mathrm{d} s\right],
$$


where

$$
C_{1}=\max \left\{\left(1+\int_{0}^{1} \tau p(\tau) b_{1}(\tau) \mathrm{d} \tau\right),\left(1+\int_{0}^{1}(1-\tau) p(\tau) b_{2}(\tau) \mathrm{d} \tau\right)\right\}
$$

Letting

$$
\tau(t)=\int_{t}^{1} e_{2}(s) h(s) \mathrm{d} s+\int_{0}^{t} e_{1}(s) h(s) \mathrm{d} s
$$

we obtain

$$
\int_{0}^{1}|\tau(t)| \mathrm{d} t \leqslant 2 \omega \int_{0}^{1} G(s, s) h(s) \mathrm{d} s<\infty .
$$

This is sufficient to ensure the relative compactness of the image $T(X)$ via the AscoliArzelà theorem.

As a result, $(2.5)$ has a solution $u \in C[0,1]$. We claim that

$$
\alpha(t) \leqslant u(t) \leqslant \beta(t) \text { for all } t \in[0,1] .
$$

Suppose that, without loss of generality, the first inequality is not true. Then there exists a $t^{*} \in(0,1)$ with $u\left(t^{*}\right)<\alpha\left(t^{*}\right)$. By continuity, we can find a maximal open interval $\left(t_{1}, t_{2}\right) \subset(0,1)$ such that $t^{*} \in\left(t_{1}, t_{2}\right)$ and

$$
u\left(t_{1}\right)=\alpha\left(t_{1}\right), \quad u\left(t_{2}\right)=\alpha\left(t_{2}\right), \quad u(t)<\alpha(t) \quad \text { for all } t \in\left(t_{1}, t_{2}\right) .
$$

For $t \in\left(t_{1}, t_{2}\right)$, we have $v^{*}(t, u(t))=v(t, \alpha(t))$ and, therefore,

$$
-u^{\prime \prime}+p(t) u=v(t, \alpha(t)) \text { for all } t \in\left(t_{1}, t_{2}\right) .
$$

On the other hand, as $\alpha$ is a lower solution of (2.3), we also have

$$
-\alpha^{\prime \prime}+p(t) \alpha \leqslant v(t, \alpha(t)) \text { for all } t \in\left(t_{1}, t_{2}\right) .
$$

Then, setting

$$
z(t):=\alpha(t)-u(t) \quad \text { for } t \in\left[t_{1}, t_{2}\right]
$$

we obtain

$$
-z^{\prime \prime}+p(t) z \leqslant 0 \text { for } t \in\left(t_{1}, t_{2}\right),
$$

with $z(t)>0$ for $t \in\left(t_{1}, t_{2}\right)$ and $z\left(t_{1}\right)=0=z\left(t_{2}\right)$. Multiplying (2.8) by

$$
G_{0}(t, s)=\frac{1}{t_{2}-t_{1}} \begin{cases}\left(s-t_{1}\right)\left(t_{2}-t\right) & \text { for } t_{1} \leqslant s \leqslant t \leqslant t_{2} \\ \left(t-t_{1}\right)\left(t_{2}-s\right) & \text { for } t_{1} \leqslant t \leqslant s \leqslant t_{2}\end{cases}
$$

and integrating both sides from $t_{1}$ to $t_{2}$ we have

$$
-\int_{t_{1}}^{t_{2}} G_{0}(t, s) z^{\prime \prime}(s) \mathrm{d} s+\int_{t_{1}}^{t_{2}} G_{0}(t, s) p(s) z(s) \mathrm{d} s \leqslant 0 .
$$


Using

$$
-\int_{t_{1}}^{t_{2}} G_{0}(t, s) z^{\prime \prime}(s) \mathrm{d} s=z(t)
$$

we have

$$
z(t)+v(t) \leqslant 0 \quad \text { for } t \in\left[t_{1}, t_{2}\right]
$$

where

$$
w(t)=\int_{t_{1}}^{t_{2}} G_{0}(t, s) p(s) z(s) \mathrm{d} s .
$$

Now, since $z(t)>0$ for $t \in\left(t_{1}, t_{2}\right)$, we have

$$
w^{\prime \prime}=-p(t) z(t)<0 \quad \text { for } t \in\left(t_{3}, t_{4}\right)
$$

and $w\left(t_{1}\right)=w\left(t_{2}\right)=0$. Thus, $w(t) \geqslant 0$ for $t \in\left(t_{1}, t_{2}\right)$, so $z(t)+w(t)>0$ for $t \in\left(t_{1}, t_{2}\right)$. This contradicts (2.9).

The proof of Theorem 1.1 follows closely the ideas in [3]. For completeness we briefly sketch the proof.

Proof of Theorem 1.1. For any $n \in N, n \geqslant 1$, let $e_{n}$ be the compact subinterval of $(0,1)$ defined by

$$
e_{n}:=\left[\frac{1}{2^{n+1}}, 1-\frac{1}{2^{n+1}}\right]
$$

From assumption (H1), there exists an $\varepsilon_{n}>0$ such that

$$
f(t, u)>L \text { for }(t, u) \in e_{n} \times\left(0, \varepsilon_{n}\right] \quad \text { and } \quad \varepsilon_{n} \leqslant \frac{L}{\max _{t \in[0,1]} p(t)}
$$

Without loss of generality (taking, if we need to, a smaller $\varepsilon_{n}$ ), we can assume that $\left\{\varepsilon_{n}\right\}$ is a decreasing sequence and $\lim _{n \rightarrow+\infty} \varepsilon_{n}=0$.

We can choose a function $\alpha \in C[0,1] \cap C^{2}(0,1)$ (see [3, p. 692]) such that

$$
\begin{aligned}
& \alpha(0)=0, \quad \alpha(1)=0, \\
& \alpha(t)>0 \text { for } t \in(0,1) \text {, } \\
& \alpha(t) \leqslant\left\{\begin{array}{ll}
\varepsilon_{1} & \text { for } t \in e_{1}, \\
\varepsilon_{n} & \text { for } t \in e_{n} \backslash e_{n-1}, n \geqslant 2 .
\end{array}\right\}
\end{aligned}
$$

Note that

$$
f(t, u) \geqslant L, \quad \forall(t, u) \in(0,1) \times\{u \in(0, \infty): 0<u \leqslant \alpha(t)\} .
$$

Set

$$
k_{0}:=\min \left\{1, \frac{L}{\left|\alpha^{\prime \prime}\right|_{\infty}+|p \alpha|_{\infty}+1}\right\} .
$$

Now we make some claims that yield the proof of the theorem. 
Claim 1. Let $h(t, u) \geqslant f(t, u)$ for $(t, u) \in(0,1) \times(0, \infty)$ with $h:(0,1) \times(0, \infty) \rightarrow$ $(0, \infty)$ a continuous function and let $v \in C[0,1] \cap C^{2}(0,1), v(t)>0$ for $t \in(0,1)$ be any solution of

$$
\begin{gathered}
-v^{\prime \prime}+p(t) v=h(t, v), \\
v(0) \geqslant 0, \quad v(1) \geqslant 0 .
\end{gathered}
$$

Then

$$
v(t) \geqslant k_{0} \alpha(t) \quad \text { for } t \in[0,1] .
$$

The proof is similar to the proof of [1, Theorem 2] and that of (2.6) in this paper. We omit it here.

We define now, for each $n \in N, n \geqslant 1$,

$$
\eta_{n}(t):=\max \left\{\frac{1}{2^{n+1}}, \min \left\{t, 1-\frac{1}{2^{n+1}}\right\}\right\} \quad \text { for } t \in(0,1)
$$

and set

$$
\tilde{f}_{n}(t, u):=\max \left\{f\left(\eta_{n}(t), u\right), f(t, u)\right\} .
$$

We find that, for each index $n, \tilde{f}_{n}:(0,1) \times(0, \infty) \rightarrow(-\infty, \infty)$ is continuous and

$$
\begin{array}{ll}
\tilde{f}_{n}(t, u) \geqslant f(t, u) & \text { for }(t, u) \in(0,1) \times(0, \infty), \\
\tilde{f}_{n}(t, u)=f(t, u) & \text { for }(t, u) \in e_{n} \times(0, \infty) .
\end{array}
$$

Hence, the sequence of function $\left\{\tilde{f}_{n}\right\}$ converges to $f$ uniformly on any set of the form $K \times(0, \infty)$, where $K$ is an arbitrary compact subset of $(0,1)$.

Next we define, by induction,

$$
\begin{aligned}
f_{1}(t, u) & :=\tilde{f}_{1}(t, u), \\
f_{2}(t, u) & :=\min \left\{f_{1}(t, u), \tilde{f}_{2}(t, u)\right\}, \\
& \vdots \\
f_{n+1}(t, u) & :=\min \left\{f_{n}(t, u), \tilde{f}_{n+1}(t, u)\right\},
\end{aligned}
$$

Each of the $f_{i}$ is a continuous function defined on $(0,1) \times(0, \infty)$. Moreover,

$$
f_{1}(t, u) \geqslant f_{2}(t, u) \geqslant \cdots \geqslant f_{n}(t, u) \geqslant f_{n+1}(t, u) \geqslant \cdots \geqslant f(t, u)
$$

and the sequence $\left\{f_{n}\right\}$ converges to $f$ uniformly on compact subsets of $(0,1) \times(0, \infty)$. We also note that

$$
f_{n}(t, u)=f(t, u) \quad \text { for }(t, u) \in e_{n} \times(0, \infty) .
$$

Consider the sequence of $B V P S$

$$
\left.\begin{array}{rl}
-u^{\prime \prime}+p(t) u & =f_{n}(t, u) \quad \text { in }(0,1), \\
u(0) & =u(1)=\varepsilon_{n} .
\end{array}\right\}
$$


Claim 2. For any $c \in\left(0, \varepsilon_{n}\right]$, the constant function $\alpha_{n}(\cdot) \equiv c$ is a lower solution of problem $(2.14)_{n}$.

It is easy to prove (i.e. it is clear once we prove (use induction), for each $t \in(0,1)$, that $c p(t) \leqslant f_{n}(t, c)$ for $\left.t \in\left(0, \varepsilon_{n}\right]\right)$, so we leave the details to the reader.

Claim 3. Any solution $u_{n}(\cdot)$ of $(2.14)_{n}$ is an upper solution of $(2.14)_{n+1}$.

Proof of Claim 3. From (2.13) we have

$$
-u_{n}^{\prime \prime}+p(t) u_{n}=f_{n}\left(t, u_{n}\right) \geqslant f_{n+1}\left(t, u_{n}\right) \text { for } t \in(0,1) .
$$

Moreover, $u_{n}(0)=u(1)=\varepsilon_{n}>\varepsilon_{n+1}$ and the conclusion follows.

Claim 4. Problem $(2.14)_{1}$ has at least one solution.

Proof of Claim 4. We fix a constant $c_{1}>\varepsilon_{1}$. From (H2) we can find a function $h_{c_{1}} \in M$ such that

$$
|f(t, u)| \leqslant h_{c_{1}}(t) \quad \text { for }(t, u) \in(0,1) \times\left(c_{1}, \infty\right) .
$$

Moreover,

$$
\left|f\left(\eta_{1}(t), u\right)\right| \leqslant h_{c_{1}}\left(\eta_{1}(t)\right) \leqslant R \quad \text { for }(t, u) \in(0,1) \times\left(c_{1}, \infty\right),
$$

where $R>c_{1} \max _{t \in[0,1]} p(t)$ is a suitable constant. Setting $q(t):=h_{c_{1}}(t)+R$, we have $q \in M$ with

$$
\left|f_{1}(t, u)\right| \leqslant q(t) \quad \text { for }(t, u) \in(0,1) \times\left(c_{1}, \infty\right) .
$$

Let $\beta \in C[0,1] \cap C^{2}(0,1)$ be the solution of the BVP

$$
\begin{gathered}
-u^{\prime \prime}+p(t) u=q(t), \\
u(0)=u(1)=c_{1} .
\end{gathered}
$$

It is easy to check that such a solution exists. We can prove (see the proof of (2.6)) that

$$
\beta(t) \geqslant c_{1} \quad \text { for } t \in[0,1]
$$

From (2.15), we have

$$
-\beta^{\prime \prime}+p(t) \beta=q(t) \geqslant f_{1}(t, \beta)
$$

and so $\beta$ is an upper solution of problem $(2.14)_{1}$.

If we now take $\alpha_{1} \equiv \varepsilon_{1}$ and recall Claim 2, we find that $\alpha_{1}$ and $\beta_{1}:=\beta$ are a lower solution and an upper solution, respectively, of problem $(2.14)_{1}$ with $\alpha_{1}(t) \leqslant \beta_{1}(t)$ for $t \in(0,1)$. Then, by Theorem 2.1 we know that there is a solution $u_{1}(\cdot)$ of $(2.14)_{1}$ such that $\varepsilon_{1}=\alpha_{1}(t) \leqslant u_{1}(t) \leqslant \beta_{1}(t)$ for $t \in(0,1)$. Claim 4 is thus proved. 
By Claim 2 and proceeding by induction using Claim 3, we obtain (via Theorem 2.1) a sequence $\left\{u_{n}(\cdot)\right\}$ of solutions to $(2.14)_{n}$ such that

$$
\begin{array}{cc}
\varepsilon_{n} \leqslant u_{n}(t) \leqslant u_{n-1}(t) & \text { for } t \in[0,1], \\
k_{0} \alpha(t) \leqslant u_{n}(t) & \text { for } t \in[0,1], \\
u_{n}(0)=\varepsilon_{n}, \quad u_{n}(1)=\varepsilon_{n} .
\end{array}
$$

We see that the series of functions $\left\{u_{j}(t)\right\}_{j=1}^{\infty}$ converges pointwise on $[0,1]$. Let

$$
u(t)=\lim _{n \rightarrow \infty} u_{n}(t) .
$$

It is clear that, for any $n \geqslant 1$,

$$
k_{0} \alpha(t) \leqslant u(t) \leqslant u_{n}(t) \text { for } t \in[0,1] .
$$

Now let $K \subset(0,1)$ be a compact interval.

There is an index $n^{*}=n^{*}(K)$ such that $K \subset K_{n}$ for all $n \geqslant n^{*}$ and, therefore, for these $n \geqslant n^{*}$,

$$
-u_{n}^{\prime \prime}+p(t) u_{n}=f_{n}\left(t, u_{n}(t)\right)=f\left(t, u_{n}(t)\right) \quad \text { for } t \in K .
$$

Hence, the function $u_{n}$ is a solution of equation (1.2) for all $t \in K$ and $n \geqslant n^{*}$. Moreover,

$$
\sup \left\{|f(t, x)|+p(t) x: t \in K, k_{0} \alpha(t) \leqslant x \leqslant u_{n^{*}}(t)\right\}<\infty .
$$

Thus, by the Ascoli-Arzelà theorem one can conclude that $u$ is a solution of (1.2) on interval $K$. Since $K$ was arbitrary, we find that

$$
-u^{\prime \prime}+p(t) u=f(t, u) \text { for } t \in(0,1) .
$$

Moreover, $u(0)=u(1)=\lim _{n \rightarrow \infty} \varepsilon_{n}=0$. One can easily prove (see [3, p. 697]) that $u$ is continuous at $t=0,1$.

Using the method in the proof of (2.6) we can easily make the following claim.

Claim 5. Suppose that, for each $t \in(0,1), f(t)$ is non-increasing. Then (1.2) has at most one solution.

\section{The proof of Theorem 1.2}

Let

$$
f^{*}(t, x)= \begin{cases}f(t, x), & x \leqslant C, \\ f(t, C), & x>C,\end{cases}
$$

and

$$
m^{*}(t, x)= \begin{cases}m(t, x), & x \leqslant C \\ m(t, C), & x>C\end{cases}
$$


Consider the BVP

$$
\left.\begin{array}{c}
-u^{\prime \prime}+p(t) u=f^{*}(t, u), \quad t \in(0,1), \\
u(0)=0=u(1) .
\end{array}\right\}
$$

Theorem 1.1 guarantees that problem (3.1) has a positive solution $u^{*} \in C[0,1] \cap C^{2}(0,1)$. Next we show that

$$
u^{*}(t) \leqslant C \quad \text { for } t \in[0,1] .
$$

Suppose that (3.2) is false. Now, since $u^{*}(0)=u^{*}(1)=0$, there exists either

(i) $t_{1}, t_{2} \in(0,1), t_{2}<t_{1}$ with $0<u^{*}(t) \leqslant C$ for $t \in\left[0, t_{2}\right), u^{*}(t)=C$ and $u^{*}(t)>C$ on $\left(t_{2}, t_{1}\right)$ with $u^{* \prime}\left(t_{1}\right)=0$, or

(ii) $t_{3}, t_{4} \in(0,1), t_{4}<t_{3}$ with $0<u^{*}(t) \leqslant C$ for $t \in\left(t_{3}, 1\right], u^{*}\left(t_{3}\right)=C$ and $u^{*}(t)>C$ on $\left(t_{4}, t_{3}\right)$ with $u^{* \prime}\left(t_{4}\right)=0$.

We can assume without loss of generality that either $t_{1} \leqslant \frac{1}{2}$ or $t_{4} \geqslant \frac{1}{2}$. Suppose $t_{1} \leqslant \frac{1}{2}$. Notice that for $t \in\left(t_{2}, t_{1}\right)$ we have

$$
\begin{aligned}
-u^{* \prime \prime} & \leqslant-u^{* \prime \prime}+p(t) u^{*} \\
& =q(t) m^{*}\left(t, u^{*}\right) \\
& =q(t) m(t, C) \\
& \leqslant q(t)[g(C)+h(C)] .
\end{aligned}
$$

Integrate (3.3) from $t_{2}$ to $t_{1}$ to obtain

$$
u^{* \prime}\left(t_{2}\right) \leqslant[g(C)+h(C)] \int_{t_{2}}^{t_{1}} q(s) \mathrm{d} s
$$

and this, together with $u^{*}\left(t_{2}\right)=C$, yields

$$
\frac{u^{* \prime}\left(t_{2}\right)}{g\left(u^{*}\left(t_{2}\right)\right)} \leqslant\left[1+\frac{h(C)}{g(C)}\right] \int_{t_{2}}^{t_{1}} q(s) \mathrm{d} s .
$$

Also, for $t \in\left(0, t_{2}\right)$ we have

$$
\begin{aligned}
-u^{* \prime \prime} & \leqslant-u^{* \prime \prime}+p(t) u^{*}=q(t) m\left(t, u^{*}\right) \\
& \leqslant q(t)\left[g\left(u^{*}(t)\right)+h\left(u^{*}(t)\right)\right],
\end{aligned}
$$

and so

$$
\begin{aligned}
\frac{-u^{* \prime \prime}(t)}{g\left(u^{*}(t)\right)} & \leqslant q(t)\left[1+\frac{h\left(u^{*}(t)\right)}{g\left(u^{*}(t)\right)}\right] \\
& \leqslant q(t)\left[1+\frac{h(C)}{g(C)}\right] \quad \text { for } t \in\left(0, t_{2}\right) .
\end{aligned}
$$

Integrate from $t \in\left(0, t_{2}\right)$ to $t_{2}$ to obtain

$$
\frac{-u^{* \prime}\left(t_{2}\right)}{g\left(u^{*}\left(t_{2}\right)\right)}+\frac{u^{* \prime}(t)}{g\left(u^{*}(t)\right)}+\int_{t}^{t_{2}}\left\{\frac{-g^{\prime}\left(u^{*}(t)\right)}{g^{2}\left(u^{*}(t)\right)}\right\}\left[u^{* \prime}(t)\right]^{2} \mathrm{~d} t \leqslant\left[1+\frac{h(C)}{g(C)}\right] \int_{t}^{t_{2}} q(s) \mathrm{d} s
$$


and this, together with (3.4) and (3.5), yields

$$
\frac{u^{* \prime}(t)}{g\left(u^{*}(t)\right)} \leqslant\left[1+\frac{h(C)}{g(C)}\right] \int_{t}^{t_{1}} q(s) \mathrm{d} s \quad \text { for } t \in\left(0, t_{2}\right) .
$$

Integrate from 0 to $t_{2}$ to find

$$
\int_{0}^{C} \frac{\mathrm{d} v}{g(v)} \leqslant\left[1+\frac{h(C)}{g(C)}\right] \frac{1}{1-t_{1}} \int_{0}^{t_{1}} s(1-s) q(s) \mathrm{d} s,
$$

i.e.

$$
\begin{aligned}
\int_{0}^{C} \frac{\mathrm{d} v}{g(v)} & \leqslant 2\left[1+\frac{h(C)}{g(C)}\right] \int_{0}^{1 / 2} s(1-s) q(s) \mathrm{d} s \\
& \leqslant b_{0}\left[1+\frac{h(C)}{g(C)}\right] .
\end{aligned}
$$

This is a contradiction, so (3.2) holds (a similar argument yields a contradiction if $t_{4} \geqslant \frac{1}{2}$ ). Thus, we have

$$
0<u^{*}(t) \leqslant C \quad \text { for } t \in(0,1), \quad u^{*}(0)=u^{*}(1)=0,
$$

so $u^{*} \in C[0,1] \cap C^{2}(0,1)$ is a positive solution of problem (1.2).

Acknowledgements. This research is supported by the National Natural Science Foundation of China (Grant no. 10301033).

\section{References}

1. R. P. AgARWAL And D. O'REgan, Singular differential and integral equations with applications (Kluwer, Dordrecht, 2003).

2. C. ARANDA And T. Godoy, On a nonlinear Dirichlet problem with a singularity along the boundary, Diff. Integ. Eqns 15 (2002), 1313-1324.

3. P. Habets And F. ZANOlin, Upper and lower solutions for a generalized Emden-Fower equation, J. Math. Analysis Applic. 181 (1994), 684-700.

4. D. O'REgAn, Theory of singular boundary value problems (World Scientific, 1994). 